\title{
Primera histerectomía robótica en México por patología ginecológica benigna, realizada en el Hospital Regional de Alta Especialidad de Zumpango
}

\author{
First robotic hysterectomy in Mexico for a benign gynecological pathology, \\ performed at the Hospital Regional de Alta Especialidad de Zumpango
}

\author{
Mariana Paola Carmona Olivares, ${ }^{\star}$ Marisol Rivera Hernández, ${ }^{*}$ Luis Roberto Gutiérrez Hernández, ${ }^{*}$ \\ Gustavo Adolfo Flores Rangel, ${ }^{*}$ Juan Roberto González Santamaría*
}

* Centro Avanzado de Cirugía Robótica del Hospital Regional de Alta Especialidad de Zumpango. Estado de México, México.

\section{RESUMEN}

Introducción: Desde su aprobación por la Food and Drug Administration (FDA) en el 2005, la cirugía asistida por robot se ha incorporado en el campo de la cirugía ginecológica. La tecnología robótica es un enfoque mínimamente invasivo, novedoso e innovador, con viabilidad demostrada en cirugía ginecológica y reproductiva. Reporte de caso: Mujer de 41 años con diagnóstico de miomatosis uterina y dolor pélvico crónico. Se reporta la primer histerectomía con conservación de anexos y neurectomía presacra laparoscópica asistida por robot en México, realizada en el Hospital Regional de Alta Especialidad de Zumpango. Conclusión: La histerectomía robótica es considerada una alternativa de abordaje quirúrgico en pacientes con patología benigna ginecológica.

Palabras clave: Cirugía robótica, histerectomía robótica, da Vinci.

\section{ABSTRACT}

Introduction: Since its approval by the FDA in 2005, robot-assisted surgery has been incorporated into the field of gynecological surgery. Robotic technology is a novel and innovative minimally invasive approach with proven feasibility in gynecological and reproductive surgery. Case report: 41-year-old patient diagnosed with uterine myomatosis and chronic pelvic pain. This report describes the first hysterectomy with preservation of adnexa and robot-assisted laparoscopic presacral neurectomy in Mexico, performed at the Regional Hospital of High Specialty of Zumpango. Conclusion: Robotic hysterectomy is considered an alternative surgical approach in patients with benign gynecological pathology.

Keywords: Robotic surgery, robotic hysterectomy, da Vinci.

\section{INTRODUCCIÓN}

La histerectomía es una de las cirugías más realizadas. Puede tener un abordaje abdominal, laparoscópico, vaginal o con asistencia de un robot. Los primeros reportes de casos de histerectomía datan del año 120 a. C. En 1988, H. Reich realizó la primera histerectomía laparoscópica dando lugar al nacimiento de una nueva técnica quirúrgica. ${ }^{1}$

En Estados Unidos, es la primera intervención quirúrgica no obstétrica en mujeres, con una distribución de $56 \%$ vía abdominal, 20\% laparoscópica, 19\% vaginal y $5 \%$ robótica. ${ }^{1}$ 
Independientemente de la vía de abordaje, en México no se cuenta con una cifra total a nivel nacional; ${ }^{1}$ por otra parte, se estima que una de cada tres mujeres ha sido histerectomizada al cumplir los 60 años; dos tercios de estos procedimientos se realizan por vía abdominal. ${ }^{2}$

Las principales indicaciones para la realización de histerectomía son: Endometriosis, hemorragia uterina anormal, masas anexiales benignas, dolor pélvico crónico en relación con adherencias secundarias a enfermedad inflamatoria pélvica o cirugía previa, cáncer de endometrio, de ovario y de cérvix estadio I. De este procedimiento, 90\% se realiza por indicaciones benignas; siendo la más frecuente, la miomatosis uterina con $62 \%$. $^{3,4}$

El Congreso Americano de Obstetras y Ginecólogos (ACOG, por sus siglas en inglés), recomienda el abordaje vaginal en la patología ginecológica benigna, por las ventajas que ofrece y la baja tasa de complicaciones.

En la actualidad, las tres vías principales para realizar una histerectomía son: Abdominal, vaginal y laparoscópica.

Con el fin de superar las limitaciones quirúrgicas de la intervención convencional laparoscópica, en el 2005 fue aprobado por la Food and Drug Administration (FDA) el Sistema Quirúrgico Da Vinci. ${ }^{5}$ Esta nueva tecnología representa una alternativa de atención quirúrgica cuando la histerectomía laparoscópica no es factible. La cirugía robótica es un procedimiento mínimamente invasivo y seguro, que ofrece ventajas tales como menor sangrado, menores complicaciones, disminución en el número de conversiones, estancia hospitalaria más corta, menor dolor posoperatorio, menor uso de analgésicos, retorno más rápido de la función intestinal y mejores resultados estéticos, así como mayor comodidad y precisión para el cirujano; sin embargo, al ser una tecnología novedosa, presenta algunas desventajas que incluyen altos costos, destreza limitada del cirujano y curva lenta de aprendizaje..$^{6-8}$

De acuerdo con los registros del Intuitive Surgical y Cyber Robotic, en México la asistencia de un robot en una intervención quirúrgica ginecológica se implementó en el año 2014, con la realización de la primera histerectomía robótica en una paciente con miomatosis uterina; esta intervención quirúrgica se llevó a cabo el 19 de mayo del 2014 en el Hospital Regional de Alta Especialidad de Zumpango.

El presente trabajo describe el procedimiento quirúrgico realizado, así como la evolución de la paciente.

\section{PRESENTACIÓN DEL CASO}

Paciente femenino de 41 años que ingresó en enero del 2014 con antecedentes de diabetes mellitus de cinco años de detección, dislipidemia mixta, una cesárea, dos legrados por aborto espontaneo y electrofulguración cervical por displasia. Antecedentes ginecoobstétricos: Menarca a los 12 años, ciclos regulares, gestas 5, partos 2, cesáreas 1 , abortos 2 .

Acudió por presentar durante un año periodos menstruales aumentados en cantidad con una duración promedio de 10 días, que llegaron a ocasionar anemia. Tratada inicialmente con hormonales, sin mejoría; refirió dismenorrea incapacitante que no mejoró con tratamiento médico.

A la exploración física, se encontró con adecuado estado general, palidez de tegumentos, abdomen sin datos de irritación peritoneal, no se palparon megalias, al tacto vaginal útero de aproximadamente $10 \times 6 \mathrm{~cm}$, anexos no palpables con leve sangrado transvaginal.

El ultrasonograma endovaginal reportó útero con ecogenicidad heterogénea a expensas de miomas de medianas dimensiones con dimensiones de $17 \times 15 \mathrm{~mm}, 15 \times 16$ $\mathrm{mm}$ y $15 \times 20 \mathrm{~mm}$ en sus ejes mayores. El útero en anteversión midió $92 \times 40 \times 61 \mathrm{~mm}$ en sus ejes mayores, resto sin lesiones; se hizo el diagnóstico de miomatosis uterina de medianos elementos (Figura 1).

La biopsia de endometrio lo reportó en estado proliferativo, Papanicolaou con alteraciones inflamatorias; se determinó riesgo quirúrgico ASA 2B, Goldman II. Fue llevada a histerectomía total con conservación de anexos y neurectomía presacra laparoscópica asistida por robot.

Durante el procedimiento quirúrgico, se colocó a la paciente en posición de Trendelenburg forzada, se realizó asepsia y antisepsia de región abdominocrural y vulvoperineal, colocación de sonda Foley y movilizador uterino López Zepeda, se creó neumoperitoneo con aguja de Veres; se colocó puerto principal umbilical de $12 \mathrm{~mm}$, se introdujo cámara, se realizó vista panorámica observando útero de $8 \times 7 \mathrm{~cm}$ adenomiósico y ovarios de $3 \times 2 \mathrm{~cm} \sin$ alteraciones macroscópicas. Se colocaron puertos acceso-

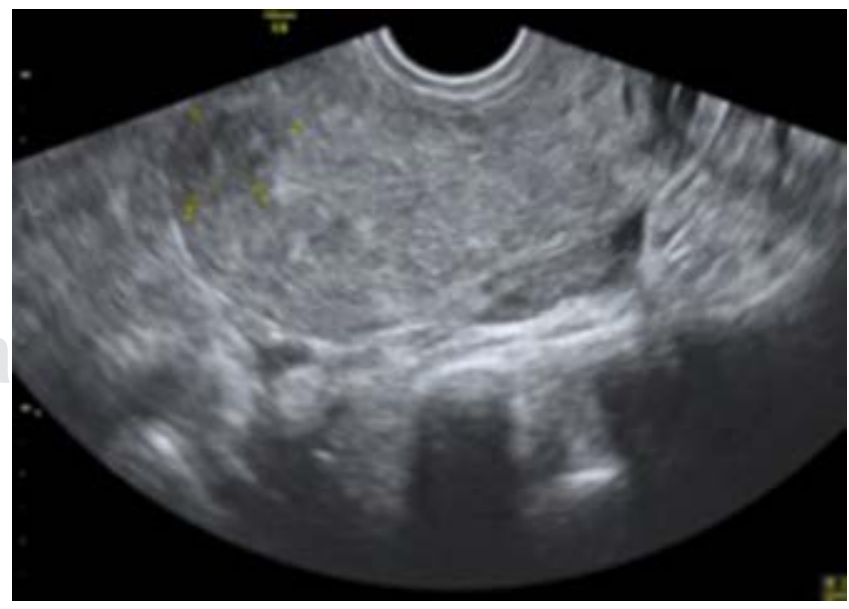

Figura 1: Ultrasonido endovaginal con ecogenicidad heterogénea a expensas de miomas de medianas dimensiones. 


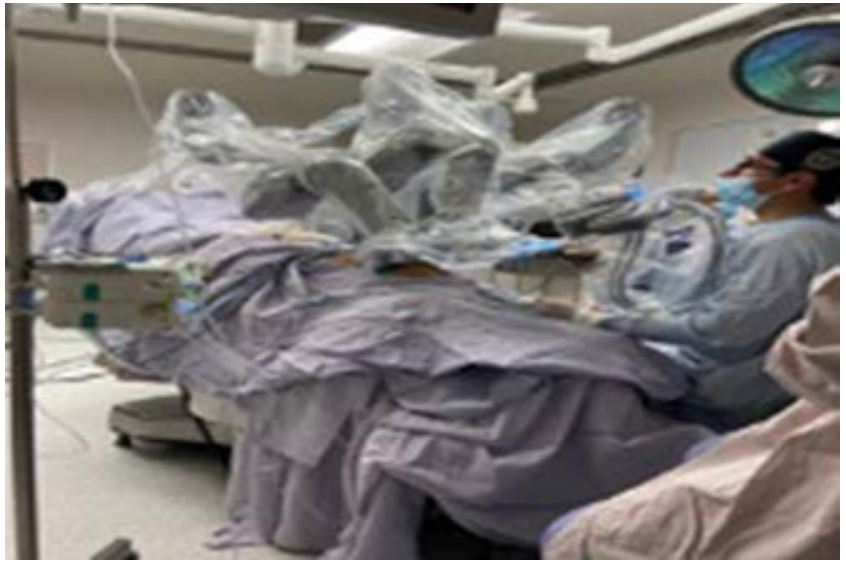

Figura 2: Posicionamiento del robot quirúrgico durante la histerectomía.

rios robóticos, uno derecho y otro izquierdo, a $10 \mathrm{~cm}$ lateral y 5 por detrás del puerto de la cámara, se realizó docking de sistema quirúrgico Da Vinci Si (Figura 2). Se procedió a realizar histerectomía con la técnica habitual (Figura 3) se extrajo pieza por vagina y se cerró cúpula vaginal con vicryl del número 1 en surgete continuo. Localizado el promontorio del sacro, se incidió en peritoneo y se ubicaron filetes de nervios presacros, los cuales se cortaron. Se finalizó la acción quirúrgica con la extracción de puertos y cierre de pared de forma convencional (Figura 4). Procedimiento sin complicaciones, sangrado $300 \mathrm{~cm}^{3}$, tiempo de docking 10 minutos, tiempo de consola $220 \mathrm{~min}$, tiempo quirúrgico $240 \mathrm{~min}$, tiempo anestésico $280 \mathrm{~min}$.

En la Unidad de Cuidados Posanestésicos se encontró con Aldrete de 9, Ramsay de 2, EVA de 0 . Al día siguiente, la paciente evolucionó a la mejoría, tolerando vía oral y presentando evacuaciones al corriente. Presentó descontrol metabólico por glucosa de $244 \mathrm{mg} / \mathrm{dL}$, por lo que se ajustó el tratamiento médico y egresó el 21 de mayo por mejoría.

El estudio histopatológico reportó útero de 147 gramos, de $9.5 \times 6 \times 5 \mathrm{~cm}$, leiomiomas uterinos intramurales $y$ adenomiosis uterina. Filetes nerviosos presacros sin alteraciones histológicas.

Se dio seguimiento convencional por consulta externa, sin complicaciones y egresó del servicio a los dos meses, por mejoría.

\section{DISCUSIÓN}

Hoy en día, existe controversia relacionada con las ventajas de la histerectomía asistida por robot respecto a las principales vías de abordaje utilizadas comúnmente. Para tratar de aclarar esta situación se han publicado estudios comparativos de las diferentes vías de abordaje quirúrgico.
La primera serie de histerectomías laparoscópicas asistidas por robot fue publicada en el 2006, Reynolds y Advincula sometieron a 16 pacientes a histerectomía laparoscópica asistida por robot. Reportaron una tasa de complicaciones del $25 \%$, la mediana del tiempo operatorio fue de 242 min (170-432) y el sangrado promedio fue de $96 \mathrm{~mL}$ (50-300 mL). ${ }^{9}$ La mediana del tiempo de estancia hospitalaria fue de 1.5 días. No hubo conversiones a laparotomía.

En el 2012, Sarlos y colaboradores realizaron un estudio comparativo del abordaje laparoscópico versus el robótico. Este estudio incluyó 100 pacientes asignadas al azar, de las cuales 95 completaron el protocolo. Los resultados obtenidos reportaron que el tiempo quirúrgico para el grupo con robot fue de 106 minutos $( \pm 29 ; 103)$ en comparación con 75 minutos $( \pm 21 ; 74)$ para el grupo convencional. La pérdida de sangre, las complicaciones, el uso de analgésicos y el retorno a la actividad para ambos grupos fueron equiparables. El cambio en el índice de calidad de vida preoperatorio a posoperatorio (calidad de vida medida en una escala lineal de 0 a 100) fue significativamente mayor en el grupo robótico, con 13 ( $\pm 10 ; 13)$ en comparación con $5( \pm 14 ; 5)$ (grupo convencional). ${ }^{10}$

El Dr. Albright y colaboradores, en el año 2016, realizaron un metaanálisis, en el cual se comparó la cirugía robótica frente a la intervención quirúrgica laparoscópica; incluyeron cuatro ensayos que cumplieron los criterios de inclusión con un total de 326 pacientes. La tasa de complicaciones fue de $12.6 \%$ para la intervención con robot y $12.8 \%$ para la cirugía laparoscópica. Las complicaciones

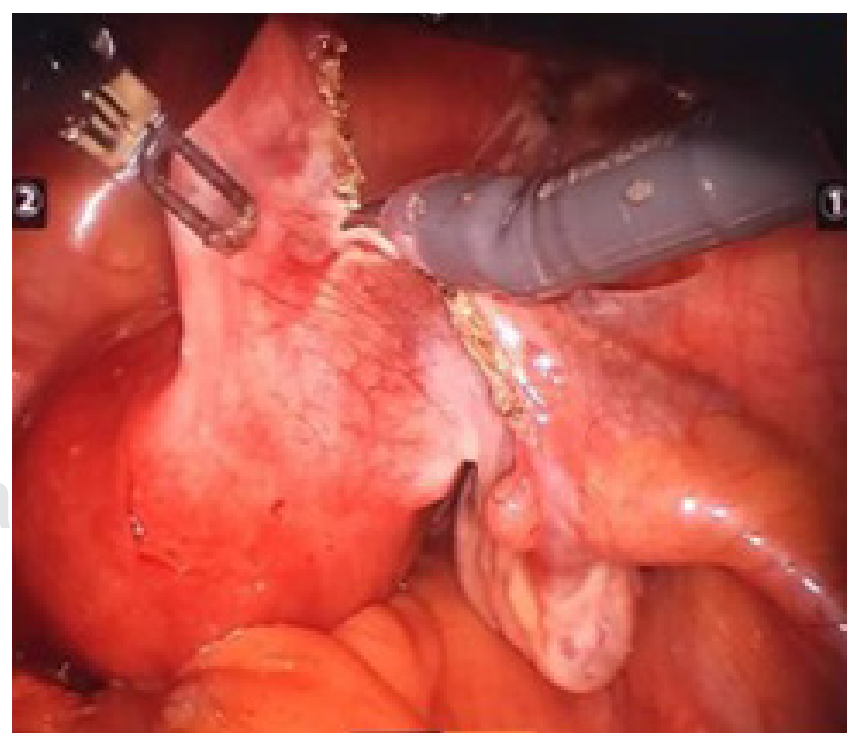

Figura 3: Corte y cauterización del ligamento infundíbulo-pélvico para liberación del cuerno uterino, con conservación de anexos. 


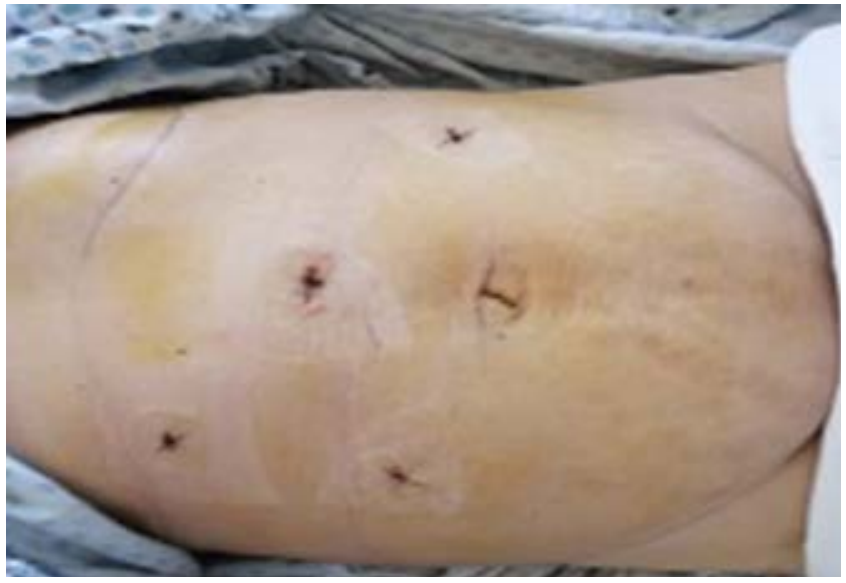

Figura 4: Posquirúrgico inmediato, donde se observan las incisiones realizadas para la colocación de los puertos.

perioperatorias más comunes fueron hematoma o sangrado del manguito vaginal $(n=12)$, dificultades robóticas que requirieron conversión a laparoscopía o cierre del manguito vaginal $(n=8)$ y transfusión de sangre $(n=4)$. En términos de duración de la estancia hospitalaria (diferencia de medias ponderada $=-0.39$ días, IC 95\% -0.92-0.14), tiempo total de operación (diferencia de medias ponderada $=9.0$ minutos, IC 95\% -31.27-47.26), conversiones a laparotomía o pérdida de sangre, el beneficio es similar en ambas técnicas quirúrgicas. ${ }^{11}$

En el 2018, en Suecia, Nina K Billfelt y colaboradores realizaron un estudio no aleatorizado con 17,806 pacientes histerectomizadas, de las cuales $54.2 \%$ fueron intervenidas por vía abdominal, $27.2 \%$ con abordaje vaginal, $11.1 \%$ por vía laparoscópica y $7.4 \%$ con operación robótica. Reportaron que la cirugía con robot presentó menor sangrado (abdominal $250 \mathrm{~cm}^{3}$, vaginal $149 \mathrm{~mL}$, laparoscópica 171 $\mathrm{mL}$, y robótica $65 \mathrm{~mL}$ ) y menos días de hospitalización (abdominal 2.5 días, vaginal 1.5 días, laparoscópica 1.6 días y con robot 1.4 días); sin embargo, la operación con robot reportó un número mayor de complicaciones, aunque menos severas (abdominal 7.6\%, vaginal 5.4\%, laparoscópica $6.6 \%$ y robótica $8.7 \%$ ). ${ }^{12}$

En México, la histerectomía es la intervención quirúrgica ginecológica realizada con mayor frecuencia. Por el área reducida y la compleja vascularidad de la pelvis este procedimiento quirúrgico implica gran atención en su realización, requiere una precisa sección, ligadura y sutura de estructuras para una meticulosa hemostasia. La cirugía robótica ofrece una alternativa hacia el abordaje quirúrgico en padecimientos ginecológicos, debido a la visión que proporciona el robot a través de un visor estéreo de alta resolución, ya que magnifica la imagen. Esto supone una ventaja muy significativa frente a la operación laparoscó- pica convencional. Por otra parte, el robot devuelve los grados de libertad perdidos en la laparoscopía, facilita la manipulación de instrumentos articulados muy pequeños y finos en la cavidad abdominal, elimina el temblor de las manos del cirujano y, por consiguiente, las operaciones son más precisas.

Como resultado de estas ventajas, se ha observado un incremento en la realización de cirugía con asistencia robótica en todo el mundo. ${ }^{13}$ En México, se realizaron 39 procedimientos ginecológicos en 2014 y en el 2017 se llegó a 214; lo cual representa un incremento del $450 \%$.

En la actualidad, la ACOG recomienda seguir realizando estudios controlados, aleatorizados y no aleatorizados, para determinar el beneficio y los riesgos potenciales de las pacientes candidatas a histerectomía con asistencia robótica.

\section{CONCLUSIONES}

La literatura en evolución sobre la cirugía asistida por robot en ginecología sugiere que las limitaciones quirúrgicas de la intervención convencional laparoscópica pueden superarse y que el nivel de destreza del cirujano puede mejorar.

La viabilidad y seguridad de la aplicación de esta tecnología está demostrada con claridad en la histerectomía. A medida que crece la experiencia, estudios prospectivos bien diseñados que comparan la cirugía asistida por robot con laparoscopía convencional y laparotomía tradicional ayudarán a caracterizar las verdaderas ventajas y desventajas de esta nueva tecnología, además de determinar aplicaciones y usos.

En el presente, la mayoría de los estudios no dan una ventaja significativa a la cirugía robótica sobre la operación laparoscópica convencional en casos benignos de enfermedad ginecológica. En contraste, la operación asistida por robot da una ventaja en intervenciones más complejas. La tasa de conversión es menor con la asistencia de un robot en comparación con el abordaje laparoscópico.

Un ejemplo de esto se observa durante una cirugía para eliminación de miomas, pues los instrumentos con tecnología EndoWrist ${ }^{\circledR}$ del aparato robótico permiten una mejor y más precisa sutura que la laparoscopía convencional con varilla recta.

Por lo tanto, la histerectomía laparoscópica asistida por robot es una nueva técnica, factible y prometedora, que puede superar las limitaciones quirúrgicas presentadas con laparoscopía convencional.

\section{REFERENCIAS}

1. Arcos Vera B, Arcos Vera D, Flores Rangel GA. Histerectomía total laparoscópica por miomatosis uterina. Rev Fac Med UNAM. 2018; 61, pp. 29-36. 
2. Murillo IJM, Pedraza GLA, Aguirre OX et al. Histerectomía por laparoscopia: experiencia de 10 años en el Hospital Español de México. Ginecol Obstet Mex. 2007; 75: 667-677.

3. Hollman-Montiel JP, Rodríguez AG. Complicaciones de la histerectomía total abdominal ginecológica por patología benigna. Arch Inv Mat Inf. 2014; 6: 25-30.

4. Guías de Práctica Clínica. Indicaciones y contraindicaciones de la histerectomía en mujeres con patología benigna en el segundo nivel de atención. 2017.

5. Villavicencio Mavrich H. Cirugía laparoscópica avanzada robótica Da Vinci: origen, aplicación clínica actual en Urología y su comparación con la cirugía abierta y laparoscópica. Actas Urol Esp. 2006; 30: 1-12. doi: 10.1016/s $\mathrm{s}^{0} 210-4806(06) 73389-7$.

6. Rojas TI, Larraín de la C D, Marengo F, González LF, Prado AJ, Buckel GH. Uso rutinario del montaje lateral (sidedocking) en cirugía robótica ginecológica: estudio de factibilidad. Rev Chil Obstet Ginecol. 2012; 77: 428-433. doi: $10.4067 / \mathrm{s}^{0} 717-75262012000600004$.

7. Advincula AP, Wang K. Evolving role and current state of robotics in minimally invasive gynecologic surgery. J Minim Invasive Gynecol. 2009; 16: 291-301. doi: 10.1016/j. jmig.2009.03.003.
8. Moreno-Portillo M, Valenzuela-Salazar C, Quiroz-Guadarrama CD et al. Cirugía robótica. Gac Med Mex. 2014; 150: 293297. doi: $10.4067 / s^{0} 718-40262012000100016$.

9. Reynolds RK, Advincula AP. Robot-assisted laparoscopic hysterectomy: technique and initial experience. Am J Surg. 2006; 191: 555-560. doi: 10.1016/j.amjsurg.2006.01.011.

10. Sarlos D, Kots L, Stevanovic N, von Felten S, Schär G. Robotic compared with conventional laparoscopic hysterectomy: a randomized controlled trial. Obstet Gynecol. 2012; 120: 604-611. doi: 10.1097/AOG.0b013e318265b61a.

11. Albright BB, Witte T, Tofte AN, Chou J, Black JD, Desai VB et al. Robotic versus laparoscopic hysterectomy for benign disease: a systematic review and meta-analysis of randomized trials. J Minim Invasive Gynecol. 2016; 23: 1827. doi: 10.1016/j.jmig.2015.08.003.

12. Billfeldt NK, Borgfeldt C, Lindkvist $H$, Stjerndahl JH, Ankardal M. A Swedish population-based evaluation of benign hysterectomy, comparing minimally invasive and abdominal surgery. Eur J Obstet Gynecol Reprod Biol. 2018; 222: 113-118. doi: 10.1016/j.ejogrb.2018.01.019.

13. Committee opinion no. 628: robotic surgery in gynecology. Obstet Gynecol. 2015; 125: 760-767. doi: 10.1097/01. AOG.0000461761.47981.07. 